Original

\title{
Necesidades de los familiares en la etapa de larga supervivencia de cáncer.
}

\author{
The needs of family members in long-term cancer \\ survivorship.
}

\author{
Beatriz Cavallar Oriol ${ }^{1}$, Cristina García-Vivar ${ }^{2,3}$ \\ 1. Clínica Universidad de Navarra. \\ 2. Universidad de Navarra, Facultad de Enfermería. \\ 3. IdiSNA, Instituto de Investigación Sanitaria de Navarra \\ Correspondencia: cgarvivar@unav.es
}

\section{RESUMEN}

Título: Necesidades de los familiares en la etapa de larga supervivencia de cáncer.

Introducción: El cáncer es una experiencia familiar en la que los supervivientes y sus familiares vivencian cambios a corto y largo plazo, especialmente en la esfera emocional. La preocupación por la recidiva - la incertidumbre del futuro generan niveles de estrés significativo en la familia. A pesar de ello, existe escasa investigación sobre las necesidades de los familiares en la supervivencia de cáncer.

Objetivos: Identificar y describir las necesidades de los familiares de pacientes supervivientes de cáncer en la etapa de larga supervivencia.

Métodos: Revisión narrativa con metodología sistemática de selección de artículos en cuatro bases de datos (PubMed, Cinhal, PsycINFO y Cochrane Library) publicados entre diciembre de 2015 y marzo de 2018, y revisión manual de 10 de las revistas más relevantes del ámbito de la oncología. Los 15 artículos identificados fueron analizados y la calidad de estos fue también evaluada.

Resultados: Los familiares, denominados como "supervivientes secundarios", tienen necesidades específicas que precisan ser abordadas por los profesionales. Estas necesidades fueron clasificadas en las siguientes categorías: apoyo emocional y espiritual, información, manejo de cuidados al superviviente $y$ atención al propio familiar.

Conclusiones: Esta revisión recopila información que demuestra que la familia debe considerarse como unidad de cuidado en la larga supervivencia de cáncer. Además, proporciona un punto de partida para el desarrollo de nuevas guías de práctica clínica que promuevan la evaluación e intervención familiar, no solo en las fases iniciales del diagnóstico y tratamiento del cáncer, sino también en la larga supervivencia de cáncer.

Palabras clave: necesidades, cuidadores, familiares, larga supervivencia, cáncer. 


\section{ABSTRACT}

Title: The needs of family members in longterm cancer survivorship.

Introduction: Cancer is a family experience in which survivors and their families experience changes in the short and long term, especially in the emotional sphere. Concerns about recurrence or the uncertainty of the future generate significant levels of stress in the family. Despite this, there is little research on the needs of family members in cancer survival.

Aims: To identify and describe the needs of family members of cancer survivors in the long-term survival.

Methods: Narrative review with systematic methodology of article selection in four databases (PubMed, Cinhal, PsyclNFO y Cochrane Library) published between December 2015 and March 2018 was conducted in addition to manual review of 10 of the most important journals in the field of oncology. The fifteen articles that met the selection criteria were analysed and the quality of these was also assessed.

Results: The family members, referred to as "secondary survivors" have specific needs that should be addressed by health professionals. These needs were classified into the following four categories: emotional and spiritual support, information, care management of the survivor, and the need for attention to the family members themselves.

Conclusions: This review compiles evidence that shows that the family should be viewed as the unit of care in the long-term cancer survivorship. Furthermore, it provides a starting point for the development of new clinical practice guidelines that promote family assessment and intervention, not only in the initial phases of cancer diagnosis and treatment, but also in the long-term cancer survivorship.

Keywords: needs, caregivers, family, longterm survivorship, cancer.

\section{INTRODUCCION}

El cáncer es una de las principales causas de morbilidad y mortalidad a nivel global, convirtiéndose en la actualidad en un problema de salud pública. Según el último informe realizado por la Organización Mundial de la Salud ${ }^{1}$ en 2012 se le atribuyeron 8,2 millones de muertes. Sin embargo, la tendencia de mortalidad por cáncer en los últimos años ha ido disminuyendo. Gracias a la investigación se han producido avances en las terapias, así como en las medidas de prevención y de diagnóstico precoz, logrando un incremento del $20 \%$ en la supervivencia en los últimos 20 años ${ }^{2}$. En España, este incremento en la supervivencia también ha sido una realidad, pues el Grupo Español de Pacientes con Cáncer (GEPAC) estima que existen hoy 1.500 .000 supervivientes de cáncer y se prevé que exista un incremento de un $50 \%$ para el año $2020^{3}$.

El concepto de supervivencia en el ámbito del cáncer fue presentado por primera vez por Fitzhugh Mullan, médico y superviviente, que diferenció tres etapas en los supervivientes del cáncer ${ }^{3}$ la supervivencia aguda (vivir con cáncer) que comienza con el diagnóstico del cáncer e incluye el período de exámenes y tratamiento del cáncer; la supervivencia extendida (intermedio; pasar el cáncer) que inicia con la remisión de la enfermedad y finalización del tratamiento inicial o mantenimiento, consolidación y seguimiento; y la supervivencia permanente (a largo plazo; vivir después del cáncer) que se identificada con la «cura»o con una supervivencia prolongada después de la remisión.

Ciertamente, el cambio en el modo de ver el cáncer- de una enfermedad aguda mortal a una enfermedad crónica- ha supuesto un cambio en la forma de abordarlo. Por ello, diferentes asociaciones nacionales $e$ internacionales han querido responder a esta realidad. Por ejemplo, la Sociedad Española de Oncología Médica ${ }^{3}$ está trabajando en un Plan Integral para la 
Asistencia al largo Superviviente de Cáncer, cuya área prioritaria es la organización de la atención a este nuevo grupo de pacientes. En esta misma línea, la Sociedad Española de Enfermería Oncológica ${ }^{4}$ está colaborando en similares iniciativas.

La evidencia disponible muestra que los largos supervivientes de cáncer a menudo tienen necesidades no cubiertas, así como dificultades en el ámbito físico, psicológico, familiar, laboral y social que pueden persistir en el tiempo o incluso ocurrir años después de finalizar el tratamiento ${ }^{3,5,6,7}$. Se han observado resultados similares en familiares de supervivientes, ya que el cáncer es una experiencia familiar en la que todos sus miembros se ven afectados ${ }^{8,9}$. Varios estudios cualitativos ${ }^{10,11}$ han señalado que los cuidadores familiares se ven afectados principalmente a nivel emocional. La preocupación por la recidiva o la incertidumbre del futuro ${ }^{9}$ les genera un estrés que en ocasiones se ve reflejado con síntomas de ansiedad, depresión y efectos psicológicos, que pueden llegar a ser significativamente mayores que en los propios supervivientes ${ }^{12,13,14,15}$. A pesar de ello, estas necesidades únicas ${ }^{16,17}$ de los cuidadores familiares de pacientes con cáncer, no son cubiertas por los profesionales de la salud.

Más aún, el cuidado en los familiares de supervivientes de cáncer se ha asociado con problemas de salud física, aumento de problemas sociales y económicos y la disminución del bienestar emocional ${ }^{17,18}$. La literatura evidencia que el cáncer sigue teniendo un impacto en la salud y el bienestar de los cuidadores familiares mucho tiempo después de finalizar el tratamiento ${ }^{19}$. Tal y como afirma Kim et al. ${ }^{20}$, los problemas y las necesidades asociadas con el cuidado informal pueden tener una duración de períodos prolongados de tiempo, incluso cuando un paciente está libre de enfermedad. Los cónyuges o miembros cercanos de la familia que se identifican como fuente primaria de apoyo del paciente superviviente de cáncer perciben escaso apoyo social, ningún reconocimiento de sus necesidades, y una menor calidad de vida ${ }^{21}$. Considerando esta realidad, algunos autores como Hodgkinson et al. $^{22}$ les denominan "segundos supervivientes" 0 "supervivientes secundarios".

\section{OBJETIVO}

Con el objetivo de conocer mejor la realidad de estos "supervivientes secundarios", el objetivo de esta revisión es identificar y describir las necesidades de los familiares en la etapa de larga supervivencia de cáncer, es decir una vez los pacientes han finalizado los tratamientos oncológicos.

\section{MÉTODOS}

Se realizó una revisión narrativa con metodología sistemática en la selección de los artículos. La búsqueda bibliográfica se llevó a cabo entre diciembre de 2015 y marzo de 2018 en las bases de datos PubMed, CINAHL, PsycINFO y Cochrane Library. Para completar la búsqueda, se revisaron manualmente 10 de las revistas más relevantes del ámbito de la oncología (Cancer Nursing, Journal of Cancer Survivorship, Oncology Nursing Forum, Psycho-oncology, Current Oncology, Oncology Nursing News, Journal of Clinical Oncology, European Journal of Oncology Nursing, European Journal of Cancer Care, Supportive Care in Cancer).

En la búsqueda se combinaron los términos: "needs", "family", "caregiver", "relative", "partner", "cancer survivor", "cancer longterm survivor", "cancer long term survivorship" utilizando los operadores booleanos AND y OR.

La búsqueda se limitó a los artículos que hacían referencia a personas adultas (de 18 años o más) y aquellos artículos publicados en inglés, castellano y francés.

\section{Criterios de selección}

Los criterios de inclusión fueron: (1) Estudios acerca de las necesidades de las 
familias de pacientes con cáncer en las etapas de supervivencia extendida o permanente; (2) Estudios comparativos sobre necesidades de los pacientes y sus familiares; (3) Estudios realizados con familias de pacientes adultos (mayores de 18 años) diagnosticados de cualquier tipo de cáncer.

\section{Búsqueda primaria}

870 artículos encontrados en las bases de datos. PubMed: 331, Cinahl: 276, Psycinfo: 261, Cochrane: 2.

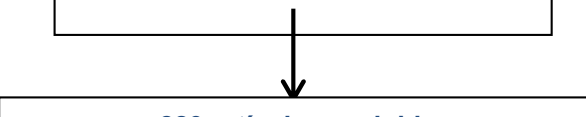

829 artículos excluidos.

PubMed: 312, Cinahl; 266, Psycinfo: 249, Cochrane:2.

Razones: artículos de la fase aguda de supervivencia o en la etapa de duelo, familiares de niños supervivientes.

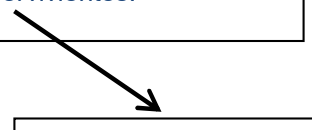

Figura 1. Diagrama de flujo PRISMA de la estrategia de búsqueda.
43 artículos a texto completo para elegibilidad

(41 de la búsqueda primaria +2 de la búsqueda secundaria).

Los criterios de exclusión fueron: (1) Artículos sobre familias de pacientes en otras etapas del cáncer (diagnóstico, tratamiento, cuidados paliativos) o familias de pacientes fallecidos de cáncer; (2) Estudios no empíricos (revisiones, casos clínicos, libros, comentarios, guías prácticas, resúmenes de conferencias o tesis de máster o doctorado).

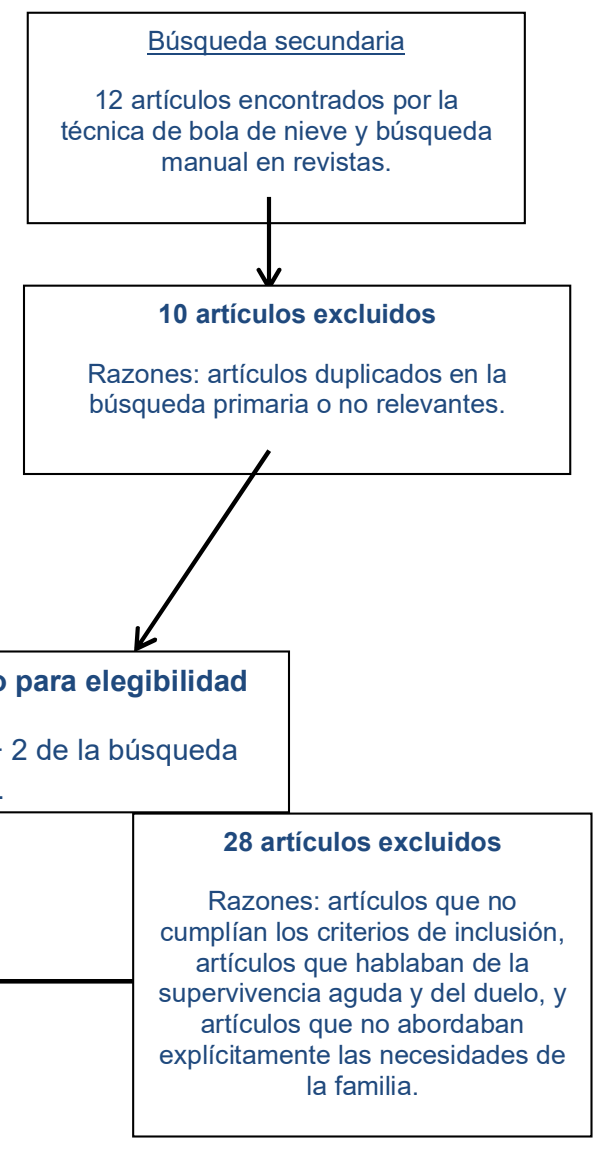

15 artículos incluidos en la revisión final

\section{Evaluación de la calidad de los artículos}

Con el objetivo de que la revisión tuviera mayor rigor metodológico, se llevó a cabo una evaluación de la calidad de los artículos seleccionados. 
La herramienta utilizada para la evaluación crítica se obtuvo de la publicación: "Instrumento para la lectura crítica y la evaluación de estudios epidemiológicos transversales" de Berra et al. (2008). El instrumento consta de un total de 27 ítems que se basa en las siguientes dimensiones: pregunta u objetivo de investigación, participantes, comparabilidad entre los grupos estudiados, definición y medición de las variables principales, análisis estadístico y confusión, resultados, conclusiones, validez externa y aplicabilidad de los resultados, y por último conflicto de intereses. Sobre cada ítem el lector anota en qué medida se cumplen los criterios («muy bien», «bien», «regular» o «mal»), o si este aspecto «no está informado» o «no aplica» para el estudio en cuestión. Finalmente, a partir de los enunciados y teniendo en cuenta los resultados obtenidos, el evaluador realiza una calificación global de la calidad de la investigación.

\section{Resultados de la búsqueda}

La búsqueda en las distintas bases de datos identificó un total de 870 artículos, de los cuales 331 eran de PubMed, 276 de Cinahl, 261 de Psycinfo y 2 de Cochrane Library. Tras la lectura del título y resumen y eliminación de duplicados se obtuvieron 41 artículos a texto completo para elegibilidad.

Mediante la técnica de "snowballing" o bola de nieve y la revisión de las revistas internacionales de oncología, se localizaron 12 nuevos artículos, de los cuales 10 fueron excluidos por ser duplicados o no relevantes. A continuación, se aplicaron los criterios de inclusión a los 43 artículos seleccionados y se procedió a la exclusión de 28 artículos que no cumplían con los criterios, y a la evaluación de la calidad de los artículos, obteniendo un total de 15 artículos para la revisión (Véase figura 1).

\section{RESULTADOS}

Esta revisión presenta las necesidades de los familiares en las etapas de supervivencia extendida y permanente. Tomando como referencia el trabajo de Hodkingson ${ }^{22}$ y Campbell ${ }^{23}$, la información analizada se agrupa en cuatro áreas: necesidad de apoyo emocional y espiritual, necesidad de información, necesidad para el manejo de cuidados al superviviente, y necesidad de atención al propio familiar (Véase tabla 1).

\section{Necesidad de apoyo emocional y espiritual}

El cuidado del cáncer es retratado como un factor de estrés que puede tener un impacto a largo plazo sobre la calidad de vida de los cuidadores, incluso después de que cesen en su rol de cuidador $^{20}$. Las preocupaciones sobre el cáncer evocados inmediatamente después del diagnóstico persisten más allá de la fase temprana de la supervivencia ${ }^{24,25}$ ya que los familiares continúan percibiendo el cáncer como una enfermedad que amenaza la vida.

Estudios han documentado los efectos secundarios negativos del cáncer en los familiares, tales como altos niveles de angustia psicológica iguales $\mathrm{o}$ incluso superiores a los de los supervivientes ${ }^{24}$. Asimismo, se han hallado problemas psicológicos persistentes en los cónyuges un año después de la finalización del tratamiento ${ }^{24}$.

De este modo, se puede observar que los familiares de supervivientes de cáncer tienen necesidades psicológicas, en particular en relación con el estrés y con hacer frente a la carga de proporcionar un cuidado físico junto con la preocupación por la salud del superviviente ${ }^{19}$. Como indican Santin et al. ${ }^{19}$, los familiares no cuentan cómo están porque toda la atención está centrada en el paciente.

Sin embargo, los familiares también requieren ayuda para gestionar las diferentes situaciones a las que se enfrentan, tales como las preocupaciones y/o miedos acerca de la recidiva del cáncer 18,21,26,27, las dificultades en la gestión de sus propias respuestas emocionales al tiempo que proporcionan apoyo a los demás ${ }^{27,29}$, 
las preocupaciones sobre el futuro de la persona que cuidan ${ }^{23,26,30}$ y ayuda para encontrar un sentido de esperanza ${ }^{23}$. Especialmente cuando se ha evidenciado que los familiares muestran angustia emocional incluso a los cincos años del diagnóstico ${ }^{25}$. $Y$ es que la percepción de estrés y amenaza se relacionan con un deterioro de la calidad de vida en el cuidador familiar ${ }^{28}$, incluso en la etapa de larga supervivencia (más de cinco años tras el diagnóstico).

Mellon et al. ${ }^{26}$ argumentan que una de las razones por las que los familiares pueden ser más susceptibles al miedo a la recidiva es porque tienen poco contacto con los profesionales de salud, y por lo tanto, menor oportunidad de recibir información directa que les ayude a disminuir sus temores. Por este motivo, las intervenciones en la supervivencia necesitan incluir programas que traten de mejorar el ajuste psicosocial y espiritual tanto del superviviente como del familiar cuidador $^{20}$ y brindar un apoyo emocional ${ }^{18}$, así como la realización de intervenciones centradas en el manejo del estrés y habilidades de afrontamiento para combatir la angustia que pueden percibir los familiares ${ }^{20}$.

\section{Necesidad de información}

La información es una de las necesidades prioritarias de los familiares en la etapa de larga supervivencia ${ }^{29,30}$. Éstos solicitan información sobre cuestiones muy diversas tales como: el diagnostico de cáncer ${ }^{25}$, los beneficios $y$ efectos secundarios del tratamiento ${ }^{27}$, el pronóstico del paciente superviviente $^{21}$, las posibilidades de recidiva $^{19}$, información sobre el riesgo familiar y consejo genético ${ }^{21}$ e información acerca de cobertura sanitaria y seguros médicos así como ayudas económicas por incapacidad laboral ${ }^{25,27,30}$.

Asimismo, otros autores destacan la necesidad de información con respecto al cuidado del superviviente en la vida diaria: las actividades beneficiosas para el superviviente, el tipo de alimentación, el manejo de los efectos secundarios en el hogar ${ }^{23}$ así como temas relacionados con la sexualidad $^{19}$.

Tal y como evidencia el estudio de Santin et al. ${ }^{19}$, la falta de información produce situaciones de ansiedad y problemas psicológicos en los cuidadores, sobre todo en los aspectos relacionados con el miedo a la recidiva del cáncer. Por este motivo autores como Harden et al. ${ }^{28}$ afirman que proporcionar a los cuidadores información temprana en el proceso de tratamiento les permitirá saber qué esperar con el tiempo. Este empoderamiento puede ayudarles a ver la prestación de cuidados de forma más positiva, y por tanto disminuir la valoración negativa de la enfermedad, logrando con ello una mejora en su calidad de vida ${ }^{28}$.

\section{Necesidad para el manejo de cuidados al superviviente}

Una de las necesidades predominantes de los familiares en el periodo de larga supervivencia hace referencia a la convivencia y manejo de los cuidados $22,25,29$. El impacto que el cáncer ha tenido sobre la relación con la persona con cáncer 17,18,27 hace que esta fase de larga supervivencia sea para los familiares de vital importancia. Por ello necesitan ayuda para saber hablar de la enfermedad, conversar abiertamente sobre el cáncer con la persona que cuidan, saber cómo reducir el estrés en su vida, en definitiva, aprender a convivir con la sombra del cáncer ${ }^{23,27}$.

Con respecto al manejo de cuidados y a la toma de decisiones en salud, Santin et al. ${ }^{19}$ destacan un déficit en la participación de los cuidadores en la toma de decisiones relacionadas con la salud del superviviente. Los cuidadores familiares tienden a sentirse excluidos de las conversaciones en las citas médicas y carecer de información sobre las consecuencias de vivir con un diagnóstico de cáncer ${ }^{19}$. Por ello, presentan la necesidad de sentirse parte de la salud del paciente junto con el equipo médico y de enfermería, y destacan la importancia de la prestación 
de una atención integral y coordinada en la etapa de supervivencia ${ }^{21}$.

\section{Necesidad de atención al propio familiar}

El cáncer y la experiencia de convivencia con el superviviente tienen un impacto en los familiares cuidadores, incluso cuando el paciente está en remisión. Se ha evidenciado una afectación en la vida de los familiares, especialmente en su funcionamiento diario, el trabajo, la salud, la espiritualidad y el futuro ${ }^{22,29}$. De este modo, los familiares identifican la necesidad de ayuda para el cuidado de su propia salud y bienestar físico y emocional $17,18,27,31$, que se concreta en recibir apoyo y asesoramiento para combatir la sensación de estrés, lograr un equilibrio en el rol de cuidador y encontrar tiempo para hacer actividades que le hagan feliz ${ }^{23,25,30}$.

A pesar de estas necesidades expresadas por los familiares, Santin et al. ${ }^{19}$ afirman que éstas no se ven satisfechas de ningún modo ya que no existe disposición formal o legal de servicios dedicados específicamente a los cuidadores de pacientes con cáncer. Los familiares perciben niveles bajos de apoyo social ${ }^{26} \mathrm{y}$ por ello consideran importante en la fase de larga supervivencia tener acceso a servicios de salud locales ${ }^{29}$, una adecuada atención integra $\left.\right|^{22,23}$ y la necesidad de prestación de apoyo por parte de la asistencia sanitaria ${ }^{19}$. De este modo, tal y como afirma Mellon et al. ${ }^{26}$, se ve necesario incluir a los supervivientes y a sus familiares en los programas de atención en la supervivencia.

Cabe destacar que las necesidades mencionadas están relacionadas con las características sociodemográficas (edad, género, raza, estatus social, etc) de los familiares cuidadores. Se confirma una asociación entre el sexo femenino y una peor calidad de vida ${ }^{19,24}$. Principalmente, las esposas son más susceptibles a los efectos negativos de la enfermedad por su mayor implicación en el papel como cuidadoras. Asimismo, las cónyuges cuidadoras muestran más restricciones en sus actividades personales y sociales, así como niveles más pobres de salud mental y mayores problemas psicológicos en comparación con los hombres que gozan de niveles más altos de calidad de vida ${ }^{26}$. Por otro lado, los cónyuges son especialmente vulnerables a una peor calidad de vida, sobre todo cuando están involucrados en la atención del cáncer a largo plazo, en comparación con los familiares cuidadores que son hijos adultos, padres o hermanos ${ }^{20}$. Los estudios también muestran que las personas de mayor edad reportan mejor salud mental o ajuste psicológico en el contexto del cuidado del cáncer, aunque presentan una mayor afectación en su salud física ${ }^{20}$.

Considerando la variabilidad de percepciones y tipos de necesidades que los familiares tienen en las distintas fases de la supervivencia, se recomienda la valoración de las características sociodemográficas del familiar cuidador y un conocimiento sobre su experiencia de convivencia con el superviviente de cáncer ${ }^{25}$.

\section{CONCLUSIONES}

Cabe señalar que la mayoría de los estudios revisados fueron de corte transversal, y por lo tanto las necesidades identificadas fueron detectadas en un determinado momento y lugar concreto. Por ello, se recomienda que futuras investigaciones se centren en el desarrollo de estudios longitudinales, con diseños mixtos que recojan la experiencia familiar a lo largo de las distintas etapas de la trayectoria del cáncer, así como estudios cualitativos que exploren y describan la experiencia familiar en la larga supervivencia de cáncer.

Esta revisión aporta luz sobre las necesidades específicas y únicas que tienen los cuidadores familiares de largos supervivientes de cáncer, tanto en la fase extendida como en la permanente. Para los familiares, el apoyo emocional y espiritual, la información y cómo manejar los cuidados del superviviente son necesidades expresadas en la etapa de larga 
supervivencia. Por ello, reclaman una atención sanitaria integral que cubra sus necesidades como "supervivientes secundarios". La familia, como unidad de cuidado, precisa una valoración y un abordaje de sus necesidades en las distintas etapas de la supervivencia. Así lo recoge también el informe sobre la "Estrategia en Cáncer del Sistema Nacional de Salud" 32 , que apunta que "es necesario abordar el impacto del cáncer, no sólo para la persona que lo padece sino también para su entorno familiar y social, en todo el proceso oncológico, incluso años después de haber concluido los tratamientos" (p.92).

Por ello, consideramos esencial que los profesionales de la salud y las instituciones se replanteen los actuales planes nacionales de atención oncológica para promover un "Plan de cuidados para el superviviente" en el que los familiares formen parte integrante de dicho plan, tal y como sucede en otros contextos internacionales ${ }^{33}, 34$. El recién publicado Plan Oncológico de Euskadi 2018-2023 35 apunta hacia la implementación de nuevos roles avanzados de las enfermeras para promover el abordaje integral y personalizado al superviviente de cáncer y su familia. Dentro de estos nuevos roles, las enfermeras de atención primaria pueden desempeñar un papel relevante en la optimización de la atención a la supervivencia y a las necesidades de las familias que conviven con el cáncer y sus secuelas ${ }^{36,37}$. Hoy más que nunca, la enfermería familiar y comunitaria, apoyada por las enfermeras especialistas en oncología, deben demostrar el impacto que tienen sus intervenciones enfermeras en la mejora de la convivencia y la calidad de vida de los largos supervivientes de cáncer y sus familiares y en la mejora de la eficiencia de tales intervenciones para el sistema sanitario.

\section{BIBLIOGRAFIA}

(1) Organización Mundial de la Salud [sede Web]. OMS, 2014 [acceso marzo de 2016] Véronique Terrasse. Informe Mundial sobre el Cáncer 2014. Disponible en: http://www.who.int/mediacentre/news/re leases/2014/cancer-report-20140203/es/

(2) Siegel RL, Miller KD, Jemal A. Cancer Statistics, 2016. Cancer J Clin. 2016; 66(1): 7-30. doi: 10.3322/caac.21332.

(3) Alba E, Jesús Cruz J, Barón F, Rodríguez C.A, Blasco A, Escobar Y, Virizuela J.A, Feliú J, Espinosa J. Monográfico SEOM de largos supervivientes de Cáncer, $1^{\underline{a}}$ parte. [monografía en Internet]. Madrid: Sociedad Española de Oncología Médica; 2012. [acceso marzo de 2016]. Disponible en: http://www.seom.org/seomcms/images/st ories/recursos/infopublico/publicaciones/ MONOGRAFICO SEOM LARGOS SUPERVI VIENTES I.pdf

(4) Sociedad Española de Enfermería Oncológica [sede Web]. Barcelona: seeo.org; 2009-2017. [acceso noviembre de 2017].

Disponible en: https://www.seeo.org/

(5) Arrigui E, Jovell AJ. Después del cáncer. Experiencias y necesidades de personas que han superado la enfermedad y de sus familiares [monografía en Internet] Barcelona: Fundació Josep Laporte; 2011. [acceso marzo de 2016]. Disponible en: http://www.seom.org/es/informacionsobre-el-cancer/publicaciones/103457despues-del-cancer-experiencias-ynecesidades-de-personas-que-hansuperado-la-enfermedad-y-de-susfamiliares

(6) Barragan B, Jovell AJ. Informe sobre las necesidades de los supervivientes de cáncer [monografía en Internet]. Grupo español de pacientes con cáncer; 2012. [acceso marzo de 2016]. Disponible en: http://www.gepac.es/images/stories/infor me supervivientes web.pdf

(7) Marzorati C, Riva S, Pravettoni G. Who Is a Cancer Survivor? A Systematic Review of Published Definitions. J Cancer Educ. 2016. doi: 10.1007/s13187-016-0997-2.

(8) Bowman KF, Rose JH, Deimling GT. Appraisal of the cancer experience by family members and survivors in long-term survivorship. Psycho-Oncology. 2006; 15: 834-45.

(9) Sklenarova H, Krumpelmann A, Haun MW, Friederich HC, Huber J, Hartmann M. When Do We Need to Care About the Caregiver? Supportive Care Needs, Anxiety, and Depression Among Informal Caregivers of 
Patients With Cancer and Cancer Survivors. Cancer. 2015; 121: 1513-19. doi: 10.1002/cncr.29223.

(10) Bishop MM, Curbow BA, Srpinger SH, Lee $J A$, Wingard JR. Comparison of lasting life changes after cancer and BMT:

perspectives of long-term survivors and spouses. Psychooncology. 2011; 20(9): 92634. doi: 10.1002/pon.1812.

(11) Adams E, Boulton M, Rose PW, Lund S, Richardson A, Wilson $S$, et al. A qualitative study exploring the experience of the partners of cancer survivors and their views on the role of primary care. Support Care Center. 2012; 20(11): 2785-94. doi: 10.1007/s00520-012-1400-4.

(12) Matthews BA. Role and gender differences in cancerrelated distress: a comparison of survivor and caregiver selfreports. Oncol Nurs Forum. 2003; 30(3): 493-99.

(13) Girgis A, Lambert S, Lecathelinais C. The supportive care needs survey for partn ers and caregivers of cancer survivors: development and psychometric evaluation. Psychooncology. 2011 ; 20(4): 387-93. doi: 10.1002/pon.1740.

(14) Mitchell AJ, Ferguson DW, Gill J, Paul J, Symonds P. Depression and anxiety in long term cancer survivors compared with spouses and healthy controls: systematic review and meta-analysis. Lancet Oncol. 2013; 14(8): 721-32. doi: 10.1016/S1470-2045(13)70244-4.

(15) Lambert SD, Girgis A, Lecathelinais C, Stacey $\mathrm{F}$. Walking a mile in their shoes: anxiety and depression among partners and caregi vers of cancer survivors at 6 and 12 months post-diagnosis. Support Care Cancer. 2013; 21(1): 75-85. doi: 10.1007/s00520-0121495-7.

(16) Lambert SD, Harrison JD, Smith E, Bonevski B, Carey M, Lawsin C et al. The unmet needs of partners and caregivers of adults diagnosed with cancer: a systematic review. BMJ Support Palliat Care. 2012; 2: 224-30. doi: 10.1136/bmjspcare-2012-000226.

(17) Girgis A, Lambert SD, McElduff P, Bonevski B, Lecathelinais C, Boyes A et al. Some things change,

some things stay the same: a longitudinal analysis of cancer caregivers' unmet supportive care needs. Psychooncology.
2013; 22(7), 1557-64. doi: 10.1002/pon.3166.

(18) Tolbert E, Bowie J, Snyder C, Bantung E, Smith K. A qualitative exploration for the experiences, needs, and roles of caregivers during and after cancer treatment: "That's what I say. I'm a relative survivor". J Cancer Surviv. 2018; 12(1): 134-44. doi: 10.1007/s11764-017-0652-x.

(19) Santin O, Treanor C, Mills M, Donnelly M. The health status and health service needs of primary caregivers of cancer survivors: a mixed methods approach. Eur J Cancer Care. 2014; 23(3): 333-39.

(20) Kim Y, Spillers RL, Hall DL. Quality of life of family caregivers 5 years after a relative's cancer diagnosis: follow-up of the national quality of life survey for caregivers. Psychooncology. 2012; 21(3): 273-81. doi: 10.1002/pon.1888.

(21) Turner $\mathrm{D}$, Adams $\mathrm{E}$, Boulton $\mathrm{M}$, Harrison $\underline{S}$, Khan N, Rose P et al. Partners and close family members of long-term cancer survivors: health status, psychosocial wellbeing and unmet supportive care needs. Psychooncology. 2013; 22(1): 12-9. doi: 10.1002/pon.2050.

(22) Hodgkinson K, Butow P, Hunt GE, Wyse R, Hobbs KM, Wain G. Life after cancer: couple's and partner's psychological adjustment and supportive care needs. Support Care Cancer. 2007; 15(4): 405-15.

(23) Campbell $\mathrm{SH}$, Sanson-Fisher $\mathrm{R}$, TaylorBrown J, Hayward L, Wang XS, Turner D. The Cancer Support Person's Unmet Needs Survey: psychometric properties. Cancer. 2009; 115(14): 3351-59. doi: 10.1002/cncr.24386.

(24) Kim Y, Spillers RL. Quality of life of family caregivers at 2 years after a relative's cancer diagnosis. Psychooncology. 2010; 19(4): 431-40. doi: 10.1002/pon.1576.

(25) Kim Y, Kashy DA, Spillers RL, Evans TV. Needs assessment of family caregivers of cancer survivors: three cohorts comparison. Psychooncology. 2010; 19(6): 573-82. doi: 10.1002/pon.1597.

(26) Mellon S, Northouse LL, Weiss LK. A population-based study of the quality of life of cancer survivors and their family caregivers. Cancer Nurs. 2006; 29(2): 12031. 
(27) Balfe M, O'Brien K, Timmons A, Butow P, O'Sullivan E, Gooberman-Hill $R$ et al. The unmet supportive care needs of long-term head and neck cancer caregivers in the extended survivorship period. J Clin Nurs. 2016; 25(11-12): 1576-86. doi: 10.1111/jocn.13140.

(28) Harden J, Sanda MG, Wei JT, Yarandi HN, Hembroff L, Hardy J, \& Northouse L. Survivorship after prostate cancer treatment: spouses quality of life at 36 months. Oncol Nurs Forum. 2013; 40(6): 567-73. doi: 10.1188/13.ONF.567-573.

(29) Hodgkinson K, Butow P, Hobbs KM, Hunt GE, Lo SK, Wain G. Assessing unmet supportive care needs in partners of cancer survivors: the development and evaluation of the Cancer Survivor's Partners Unmet Needs Measure (CaSPUN). Psychooncology. 2006; 16(9): 805-13. doi: 10.1002/pon.1138.

(30) Campbell SH, Carey M, Sanson-Fisher $\underline{\mathrm{R}}$, Barker D, Turner D, Taylor-Brown J et al. Measuring the unmet supportive care needs of cancer support persons: the development of the support person's unmet needs survey--short form. Eur J Cancer. 2013; 23(2): 255-62. doi: 10.1111/ecc.12138.

(31) Lambert S, Girgis A, Descallar J, Levesque $J V$, Jones B. Trajectories of mental and physical functioning among spouse caregivers of cancer survivors over the first years following the diagnosis. Patient Educ Couns. 2017; 100(6): 1213-21. doi: 10.1016/j.pec.2016.12.031.

(32) Borrás Andrés JM. Estrategia en Cáncer del Sistema Nacional de Salud, 2010 [monografía en Internet]. Madrid: Ministerio de Sanidad y Política Social; 2010. [acceso mayo de 2016]. Disponible en:

http://www.msssi.gob.es/organizacion/sns /planCalidadSNS/pdf/ActualizacionEstrateg iaCancer.pdf
(33) Instituto Nacional del Cáncer (NIH). Una transición difícil: los planes para supervivientes de cáncer se afianzan con lentitud. 2012. [acceso mayo de 2017]. Disponible en: http://www.cancer.gov/espanol/cancer/so brellevar/investigacion/planessupervivencia.

(34) Nekhlyudov L, Ganz PA, Arora NK, Rowland JH. Going Beyond Being Lost in Transition: A Decade of Progress in Cancer Survivorship. J Clin Oncol. 2017 20;35(18):1978-81. doi: 10.1200/JCO.2016.72.13

(35) Departamento de Salud del Gobierno Vasco. Plan Oncológico de Euskadi 20182023. Vitoria: Departamento de Salud, Osakidetza; 2018.

(36) Corcoran S, Dunne M, McCabe MS. The Role of Advanced Practice Nurses in Cancer Survivorship Care. Semin Oncol Nurs. 2015;31(4):338-347.

doi:10.1016/j.soncn.2015.08.009

(37) Luctkar-Flude M, Aiken A, McColl MA, Tranmer J. A comprehensive framework and key guideline recommendations for the provision of evidence-based breast cancer survivorship care within the primary care setting. Fam Pract. 2015;32(2):129-140. doi:10.1093/fampra/cmu082 